\title{
Evaluation of the Impact of a New Synthetic Vitamin E-Bonded Membrane on the Hypo-Responsiveness to the Erythropoietin Therapy in Hemodialysis Patients: A Multicenter Study
}

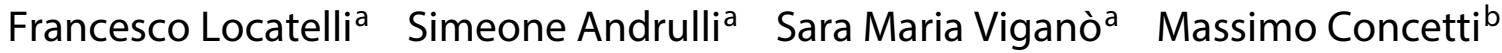 \\ Sauro Urbinic ${ }^{c}$ Franca Giacchino $^{d}$ Roberto Broccolie Filippo Aucella ${ }^{f}$ \\ Maria Cossu ${ }^{g}$ Paolo Conti ${ }^{\text {h }}$ Laura Fattori $^{i}$ Giorgio Punzol Daniela Angelini ${ }^{j}$ \\ Marco Peruzzini $^{k}$ Salvatore Di Giulio ${ }^{m}$ Marta Piroddi ${ }^{n} \quad$ Francesco Galli $^{n}$ \\ Lucia Del Vecchio ${ }^{a}$

\begin{abstract}
a Departments of Nephrology and Dialysis of Alessandro Manzoni Hospital, Lecco, ${ }^{b}$ Ospedale Civile Augusto Murri, ASUR Marche Zona Territoriale 11, Fermo, ' Ospedale G.B. Morgagni, L. Pierantoni, Forlì, dOspedale Civile, Ivrea, ${ }^{\text {e Presidio }}$ Ospedaliero Di Valle Camonica, Esine, ${ }^{f}$ Casa Sollievo della Sofferenza, San Giovanni Rotondo, ${ }^{9}$ Ospedale SS Annunziata, Sassari, hOspedale Misericordia, ASL Toscana Sud-Est, Grosseto, 'Ospedale di Senigallia, Senigallia, ${ }^{j}$ Ospedale Campo di Marte, Lucca, ${ }^{\circ}$ Ospedale Geriatrico - INRCA, Ancona, 'Ospedale Sant'Andrea, and m Ospedale Forlanini, Roma, and nLaboratory of Clinical Biochemistry and Nutrition, Department of Pharmaceutical Sciences, University of Perugia, Perugia, Italy
\end{abstract}

\section{Keywords}

Erythropoietin · Erythropoiesis-stimulating agent resistance - Erythropoiesis-stimulating agent responsiveness · Vitamin $\mathrm{E} \cdot$ Hemodialysis

\begin{abstract}
Background: Oxidative stress has been related to hypo-response to erythropoiesis-stimulating agents (ESAs) in hemodialysis (HD) patients. The aim of this study was to verify whether vitamin $\mathrm{E}$ (ViE) on a synthetic polysulfone dialyzer can improve ESA responsiveness. Methods: This controlled, multicenter study involved $93 \mathrm{HD}$ patients on stable ESA therapy, who were randomized to either ViE-coated polysulfone dialyzer or to a low-flux synthetic dialyzer. The primary outcome measure was the change in ESA resistance index (ERI) from baseline. Results: Mean ERI decreased in the ViE group by $1.45 \mathrm{IU} / \mathrm{kg}^{*} \mathrm{~g} / \mathrm{dl}$ and increased in the control group
\end{abstract}

by $0.53 \mathrm{IU} / \mathrm{kg}^{*} \mathrm{~g} / \mathrm{dl}$, with a mean difference of $1.98 \mathrm{IU} / \mathrm{kg}^{*} \mathrm{~g} / \mathrm{dl}$ $(p=0.001$ after adjusting for baseline ERI, as foreseen by the study protocol). Baseline ERI was inversely related to its changes during follow-up only in the control group $\left(R^{2}=\right.$ 0.29). Conclusions: The ViE dialyzer can improve ESA response in HD patients. Changes in ERI during follow-up are independent from baseline ERI only in the ViE group.

Video Journal Club 'Cappuccino with Claudio Ronco' at http://www.karger.com/?doi=453442.

(C) 2017 S. Karger AG, Basel

\section{Introduction}

Oxidative stress is commonly observed in hemodialysis (HD) patients, resulting from an imbalance between overproduction of reactive oxygen species and impairment of defense mechanisms $[1,2]$. Oxidative stress is

\section{KARGER}

(c) 2017 S. Karger AG, Basel

E-Mail karger@karger.com

www.karger.com/bpu
Prof. Francesco Locatelli

Department of Nephrology and Dialysis

Ospedale A. Manzoni, ASST Lecco

Via dell'Eremo 9/11, IT-23900 Lecco (Italy)

E-Mail f.locatelli@asst-lecco.it 
implicated in the development of long-term complications in HD patients and it appears as a major component of the malnutrition inflammation complex syndrome [3]. The dialysis procedure further exposes HD patients to inflammation and oxidative stress through the contact with the dialysis membrane and dialysate. Loss of low molecular weight antioxidants and activation of leukocytes may also have a contributory role.

Many factors are involved in anemia of HD patients, including a relative decrease in erythropoietin production, iron deficiency and chronic inflammation [4]. In particular, pro-inflammatory cytokine and uremic toxins may influence its severity and the ability of some patients to respond to erythropoiesis stimulating agents (ESA). Given that pro-inflammatory cytokines also worsens oxidative stress, the latter may have an independent negative role on anemia and ESA responsiveness $[5,6]$.

HD therapies aimed at reducing oxidative stress could be then of clinical relevance. The prevention of oxidative stress linked to the bio-incompatibility of the dialysis procedure may be obtained with either the supplementation of antioxidant vitamins [7] or by using vitamin $\mathrm{E}$ (ViE)-coated dialyzers $[8,9]$. Given the link between chronic inflammation, oxidative stress and anemia pathogenesis, it has been hypothesized that high-flux HD using a biocompatible ViE membrane may improve anemia and ESA response [10].

Accordingly, some years ago, we performed a crossover, randomized, pilot study, which suggested a possible positive effect of polysulfone $\mathrm{ViE}$ membrane dialyzer on ESA resistance [11].

This multicenter, randomized, controlled trial is aimed at testing whether the same synthetic polysulfone dialyzer bonded with alpha-tocopherol can improve the ESA resistance index (ERI) of HD patients, confirming and eventually expanding the results of our previous pilot study [11].

\section{Patients and Methods}

\section{Study Design}

We conducted a multicenter, controlled, randomized trial of 2 parallel groups of HD patients from 13 sites in Italy between December 2009 and April 2012. The study follow-up ended on April 2013. After giving their written informed consent, the patients were randomly assigned to the experimental treatment (high-flux polysulfone dialyzer with $\mathrm{ViE}$ ) or to the control treatment (any low-flux synthetic biocompatible dialyzer in use at the participating centers) for 12 months. According to the protocol, administration of iron and ESA should be kept constant during the study as much as possible and, eventually, changes in ESA and iron dosages should follow the suggestions of the European Renal Best Practice [12].

\section{Inclusion Criteria}

Patients of both genders aged more than 18 years were considered eligible for the study if they had been receiving bicarbonate HD for at least 6 months and were on ESA therapy with stable doses for at least 3 months. ESA hypo-responsiveness was defined as above 8 UI of epoetin/ $/ \mathrm{kg}^{*} \mathrm{~g}$ of hemoglobin $(\mathrm{Hb})$ per week. This cutoff value was obtained from the findings of our pilot study [11].

\section{Exclusion Criteria}

Patients were excluded from the study if they had had one of the following conditions in the previous 3 months: acute infections, vascular access thrombosis, acute myocardial infarction, stroke, clinically relevant bleedings, central catheter vascular access, major surgical procedures, blood transfusions, hemoglobinopathies, active malignancies and participation to other experimental clinical studies. Patients with inadequate iron stores (ferritin levels $<200$ $\mathrm{ng} / \mathrm{ml}$ and transferrin saturation $<20 \%$ ) were also excluded.

\section{Primary End Point}

ERI changes in the HD patients treated with the ViE dialyzer (experimental treatment) in comparison with those of the HD patients treated with a low-flux synthetic dialyzer (control treatment). According to the conclusions of our pilot study [11], this crude analysis was corrected for baseline ERI values.

\section{Sample Size Calculation}

The sample size was calculated considering the ERI changes during follow-up as the main response variable, and their reduction with the $\mathrm{ViE}$ experimental treatment compared to the control treatment. A 33\% reduction in the relationship with the phenomenal variability was considered as clinically relevant. The calculated sample size of 105 patients was based on a significance level ( $\alpha$ error) of 0.05 , a $\beta$ error of 0.2 , a power $(1-\beta)$ of 0.8 and an estimated drop-out rate of $10 \%$.

\section{Clinical and Laboratory Data}

At the beginning of the study, the patients were randomly assigned to the treatment dialyzer with a 1:1 ratio by means of a computer-generated random list. The dialyzer and the dialysis prescription had not been changed during the study. Pre- and postdialysis body weight, blood pressure and heart rate were recorded at baseline and every 4 months. All the drugs administered during each dialysis session and inter-dialysis therapies were also recorded.

Pre-dialysis levels of $\mathrm{Hb}$, parameters of iron status, serum albumin and parathyroid hormone (PTH) were recorded at baseline and every 4 months. In order to estimate the dialysis dose for low molecular weight uremic toxins, the intradialytic reduction of blood urea was recorded and KT/V was calculated. ERI was defined by the ratio of ESA dose per week by the body weight in $\mathrm{kg}^{*} \mathrm{Hb}$ in $\mathrm{g} / \mathrm{dl}$.

\section{Statistical Analysis}

The data were described using median values and interquartile ranges (IQRs) based on 25th and 75th percentiles for the distributed continuous variables. The baseline distribution of the con- 
Table 1. General characteristics of the analyzed patients at baseline

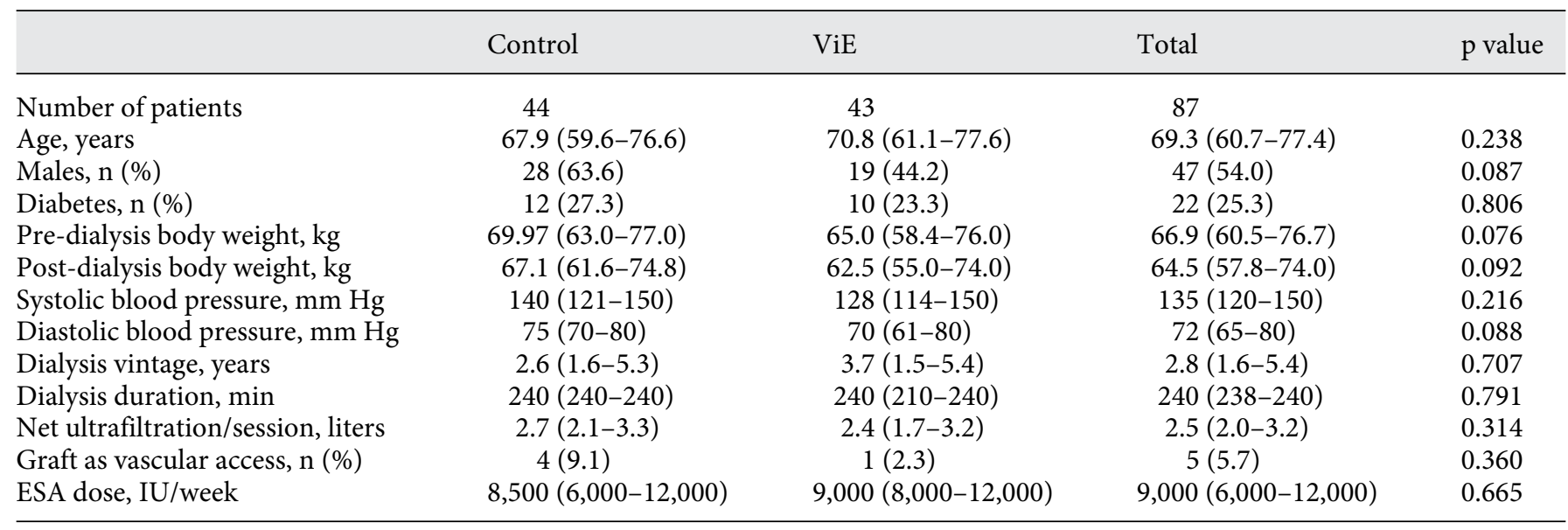

Values are median (IQR) or n (\%).

tinuous and categorical variables by group was investigated using the Mann-Whitney and chi-square tests, respectively.

The follow-up data were analyzed using the multivariate general linear model and the approach of the last available data per patient.

The primary outcome variable was the intra-patient difference over time in ERI between the last available value and the baseline one. The primary contrast of interest was the difference in ERI over time between the experimental and the control treatment groups. The multivariate analysis was planned in order to identify predictors associated with ERI and the possible additional net effect of the experimental treatment. The effect of the experimental treatment over time was tested by means of group interaction, with a hierarchical backward approach with a $p_{\text {in }}$ value of 0.1 and a $p_{\text {out }}$ value of 0.05 .

Linear regression and adjusted $\mathrm{R}$ square statistics were used to estimate the variability of ESA resistance difference over follow-up explained by some covariates, such as the baseline individual values.

All these analyses were made using SPSS statistical software for Windows, release 20.0.

The protocol was approved by the Ethics Committee of the Coordinating Centre (Alessandro Manzoni Hospital, Lecco, Italy).

\section{Results}

\section{General Characteristics}

The study involved 13 centers in Italy. Among the patients receiving chronic HD at the participating centers, 94 patients were enrolled and randomized to either the experimental treatment with the ViE-dialyzer ( 48 patients) or to the control treatment with low-flux synthetic dialyzer (46 patients). Fifty-seven patients (61\%) ended the planned 12-month follow-up period. The reasons for study discontinuation were lost to follow-up $(\mathrm{n}=17)$, death $(\mathrm{n}=10,4$ in the experimental group and 6 in the control group), kidney transplantation $(n=4)$ and other reasons $(n=6)$. None of the deaths were related to the study treatment. The distribution of followup values between groups was not statistically different $(\mathrm{p}=0.274)$ indicating that the drop-out rate was comparable in the 2 groups. Eight-seven patients out of the 94 randomized ones (93\%, 43 patients in the ViE group and 44 patients in the control group) had a follow-up of at least 4 months and represent the basis of the main analysis of the study.

Their general and biochemical characteristics at baseline are shown in tables 1 and 2 . The patients had a median age of 69.3 years and had a median dialysis vintage of 2.8 years. There were no significant differences between the 2 groups for all reported variables, with a good match for diabetes, dialysis vintage, dialysis duration, ESA dose, anemia parameters, albumin and PTH values at baseline. A little random imbalance in gender distribution was observed between the 2 groups, with a higher percentage of men in the control group (64 vs. $44 \%$, respectively; $\mathrm{p}=$ 0.087). As expected, this was associated with a little but not significant imbalance for pre- and post-dialysis body weight and pre-dialysis plasma creatinine values. Albumin and pre-/post-dialysis body weight mean values did not change significantly during the study in both groups.

The majority of the patients were treated with shortacting ESA (77\% of the patients) given intravenously. Long-acting drugs such as darbepoetin alfa (16\%) and methoxy-polyethylene glycol-epoetin beta (7\%) were used less frequently. 
Table 2. Biochemical characteristics of the patients at baseline

\begin{tabular}{|c|c|c|c|c|}
\hline Number of patients & 44 & 43 & 87 & \\
\hline Pre-dialysis creatinine, $\mathrm{mg} / \mathrm{dl}$ & $9.6(8.6-11.1)$ & $8.9(7.6-10.1)$ & $9.2(8.0-10.3)$ & 0.079 \\
\hline Post-dialysis urea, mg/dl & $48(33-58)$ & $39(30-52)$ & $42(32-55)$ & 0.175 \\
\hline $\mathrm{Hb}, \mathrm{g} / \mathrm{dl}$ & $11.1(10.6-11.9)$ & $11.2(10.6-11.9)$ & $11.2(10.6-11.9)$ & 0.668 \\
\hline Transferrin saturation, $\%$ & $22(17-33)$ & $23(19-32)$ & $22(19-32)$ & 0.568 \\
\hline Albumin, g/dl & $3.80(3.47-4.09)$ & $3.70(3.40-4.10)$ & $3.75(3.42-4.10)$ & 0.439 \\
\hline PTH, pg/ml & $147(73-265)$ & $191(98-371)$ & $185(78-287)$ & 0.548 \\
\hline ERI, IU/kg*g of $\mathrm{Hb} /$ week & $11.5(8.3-15.4)$ & $12.8(9.3-19.3)$ & $12.7(8.6-17.4)$ & 0.306 \\
\hline C-reactive protein, $\mathrm{mg} / \mathrm{dl}$ & $0.8(0.5-1.7)$ & $0.7(0.5-1.2)$ & $0.7(0.5-1.5)$ & 0.642 \\
\hline Serum phosphate, $\mathrm{mmol} / \mathrm{l}$ & $1.82(1.43-2.26)$ & $1.80(1.20-2.50)$ & $1.80(1.30-2.31)$ & 0.939 \\
\hline Urea ratio & $0.30(0.25-0.35)$ & $0.28(0.22-0.34)$ & $0.29(0.23-0.34)$ & 0.348 \\
\hline
\end{tabular}

Values are median (IQR) or $\mathrm{n}(\%)$.

Fig. 1. Evolution of $\mathrm{Hb}$ levels from baseline to the end of the follow-up in the 2 study groups (87 patients).

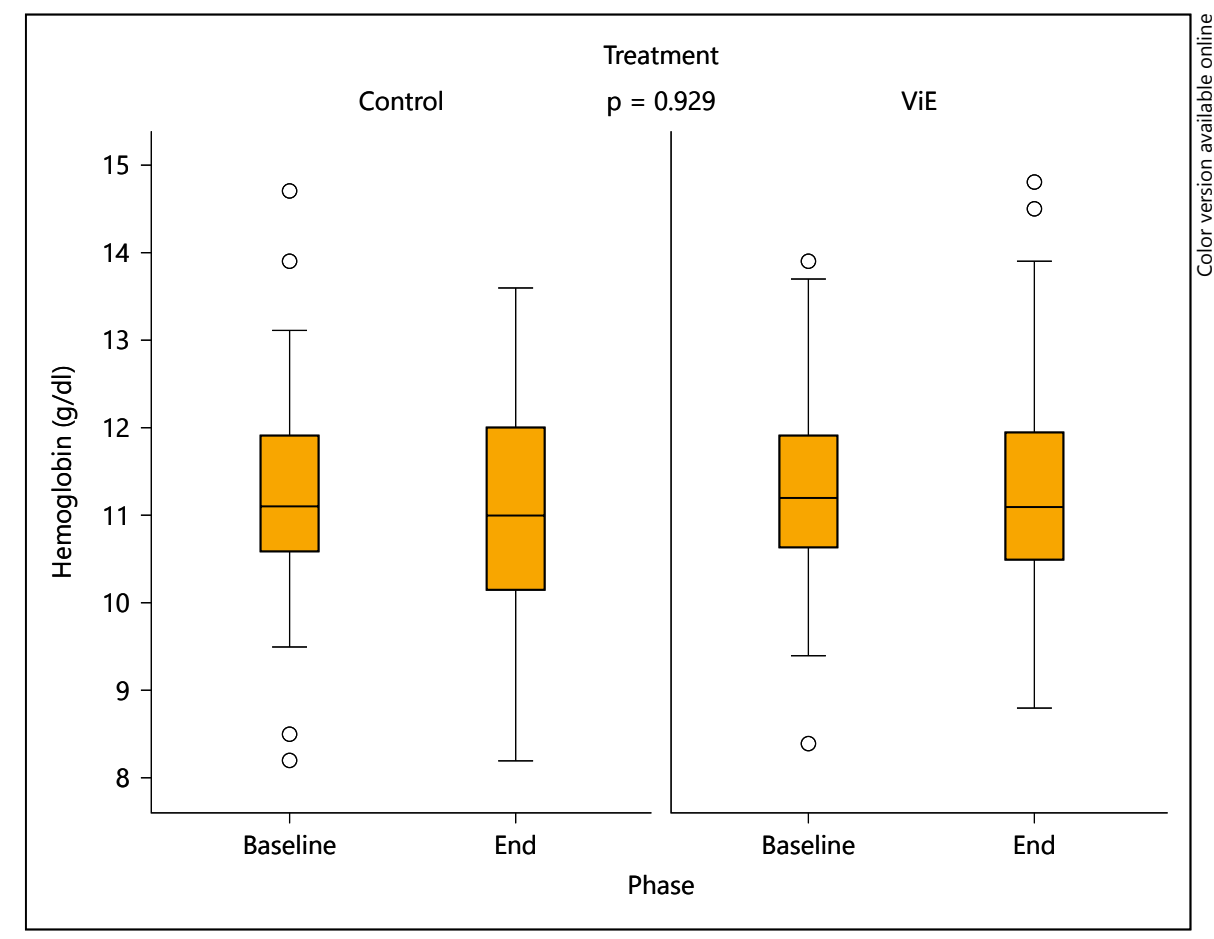

\section{Anemia Parameters and ERI during Follow-Up}

The trend of $\mathrm{Hb}$ levels in the 2 study groups is shown in figure 1 . There were no significant differences between the 2 groups at baseline $(\mathrm{p}=0.668)$ and during the follow-up $(\mathrm{p}=0.929)$. The ferritin and PTH levels remained stable in the $\mathrm{ViE}$ experimental group and tended to increase in the control group without reaching statistical significance $(p=$
0.246 and $p=0.525$, respectively; fig. 2 ). The transferrin saturation levels remained stable during the study without differences between groups $(25.8 \pm 10.2 \%$ to $27.5 \pm 13.0 \%$ in the experimental group and $24.3 \pm 10.1 \%$ to $26.4 \pm 10.7 \%$ in the control group, respectively, $\mathrm{p}=0.341$ ).

Intravenous iron therapy was administrated to the majority of the patients (53 of 87 patients; $61 \%$ ) without 


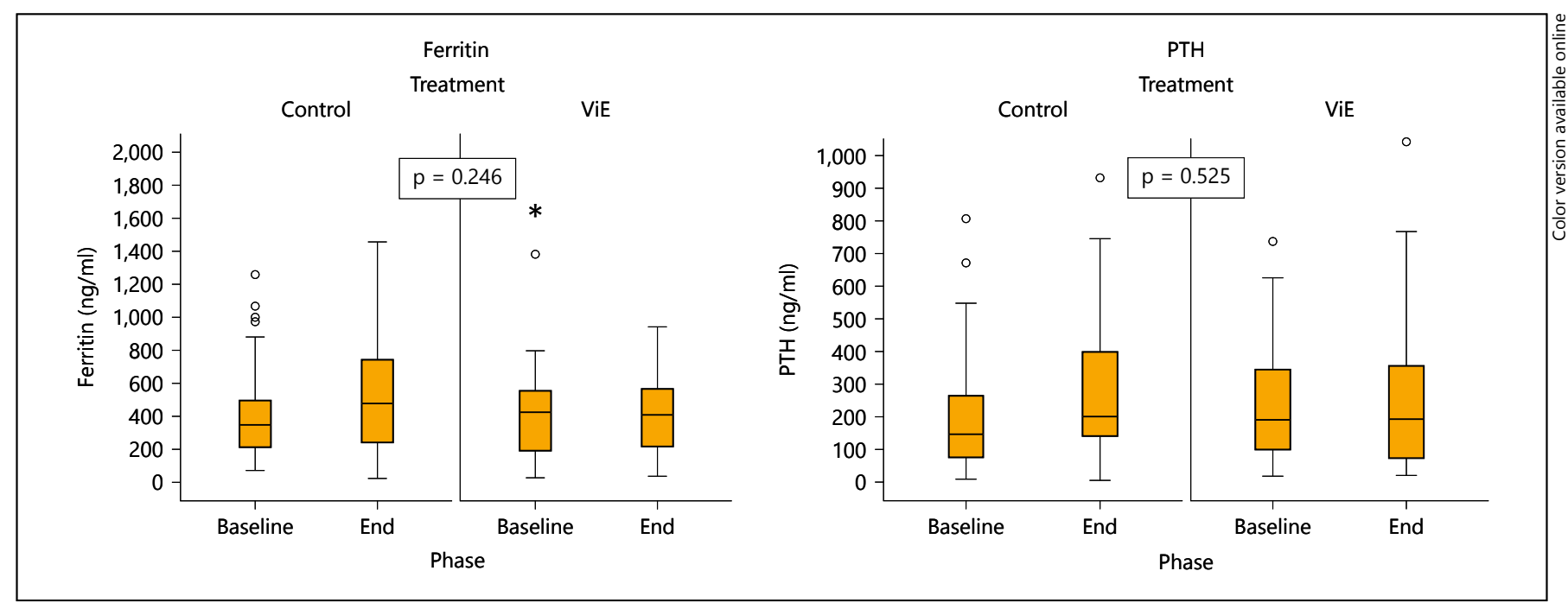

Fig. 2. Evolution of ferritin and PTH levels from baseline to the end of the follow-up in the 2 study groups (87 patients). Note that the 2 variables have a similar pattern.

Fig. 3. Evolution of ERI values from baseline to the end of the follow-up in the 2 study groups ( 87 patients). The ERI values tend to increase in the control group and to decrease in the ViE experimental group.

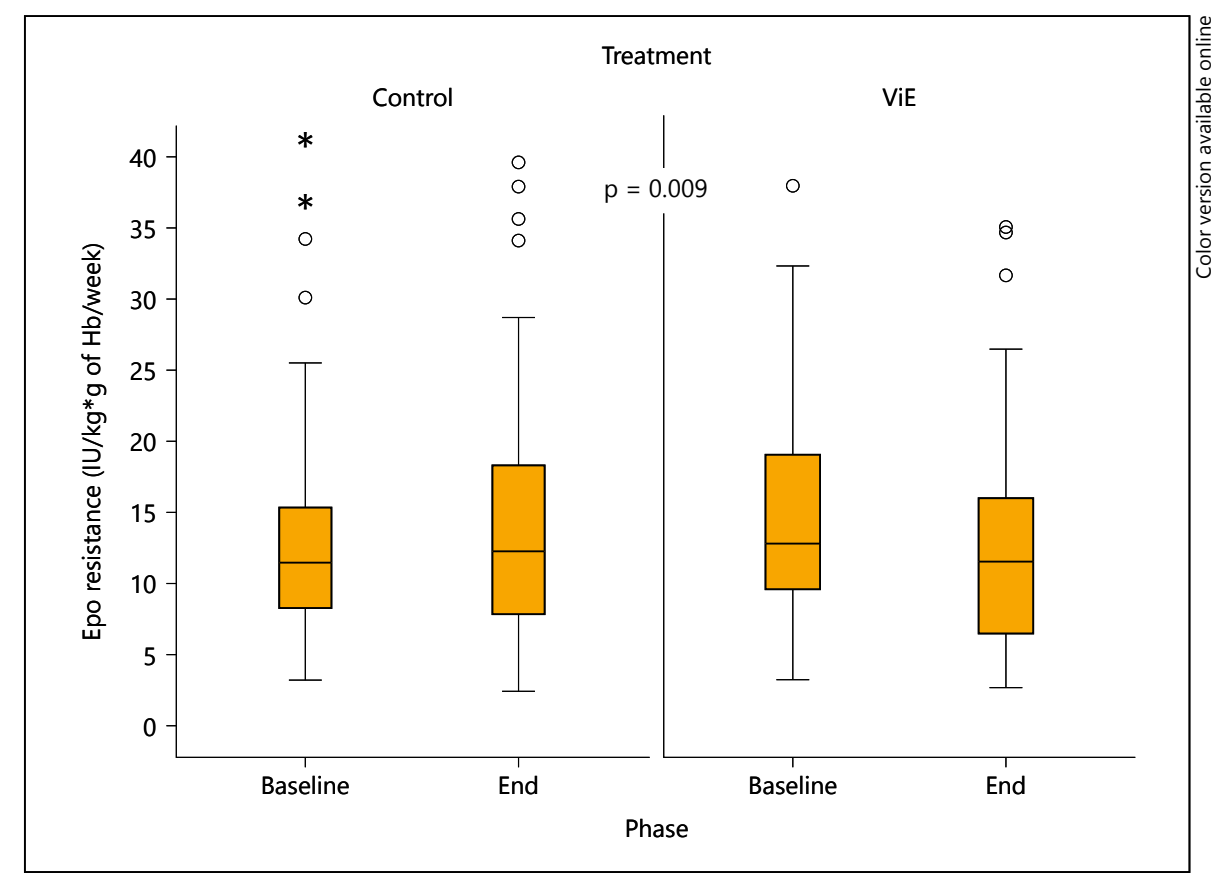

statistical differences between groups (65 and 57\% in experimental and control groups, respectively; $p=0.512$ ) and a constant dose was maintained during the study at a median dosage of $62.5 \mathrm{mg} /$ week in both groups as iron gluconate $(77 \%)$ or saccarate $(23 \%)$.

In the control group, median ESA dose tended to increase during the follow-up (from 130.5, IQR 96.4177.5 UI/kg/week to 141.7 , IQR 88.2-213.5 UI/kg/week, $\mathrm{p}=0.641$ ), while it remained stable the $\mathrm{ViE}$ group (from 139.5, IQR 108.5-222.72 UI/kg/week to 133.3, IQR 69.6$171.4 \mathrm{UI} / \mathrm{kg} /$ week).

The ERI values at baseline and during follow-up in the 2 study groups are shown in figure 3 . At baseline median ERI values tended to be higher in the $\mathrm{ViE}$ group compared to the control one (12.8 and $11.5 \mathrm{IU} / \mathrm{kg}^{*} \mathrm{~g}$ of $\mathrm{Hb} /$ week, respectively; $\mathrm{p}=0.306$ ). During follow-up, these values de- 
Fig. 4. Inverse relationship between baseline ERI values and their following variations in the 2 study groups ( 87 patients).

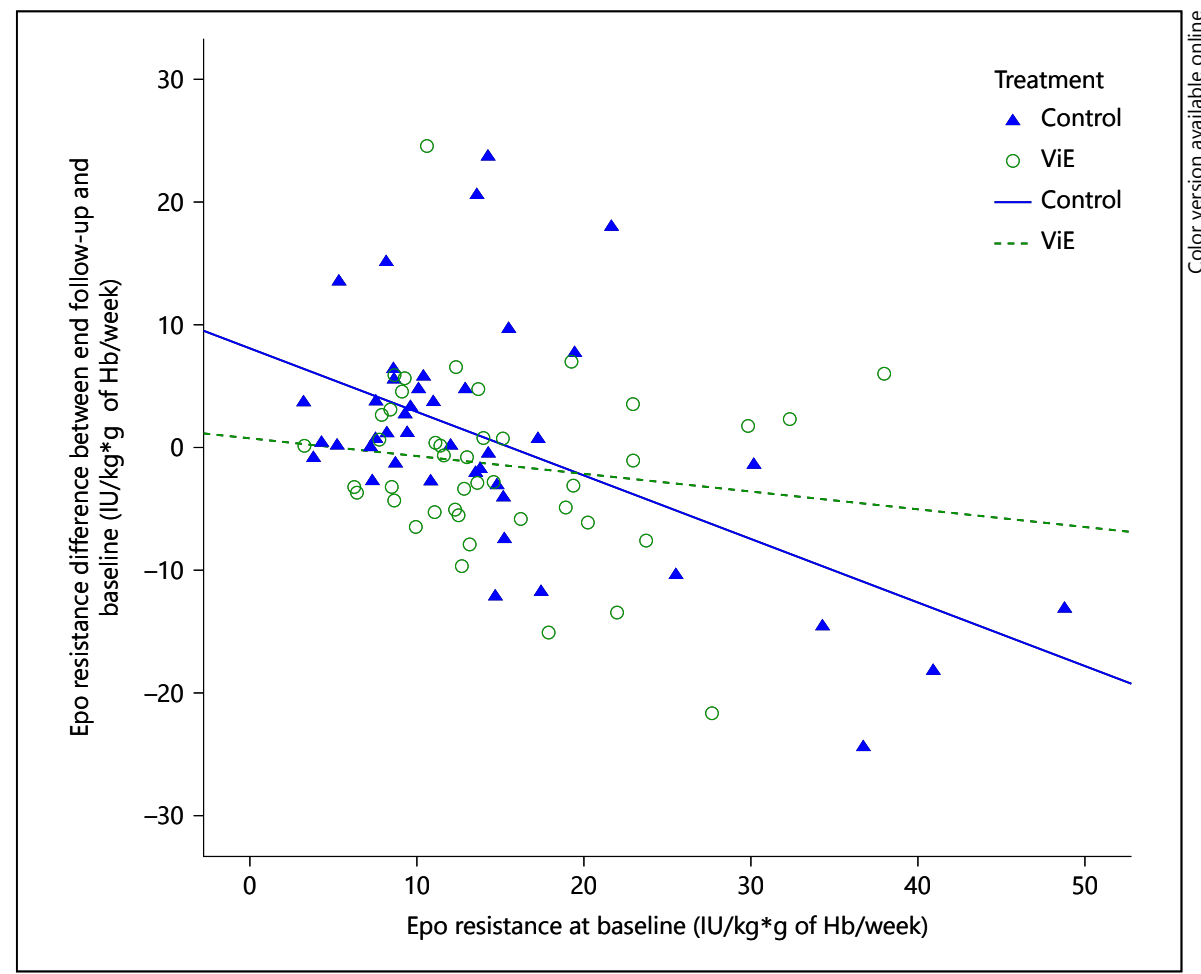

Table 3. Final model of predictors related to the ERI variation during follow-up in the 2 study groups. Dependent variable: difference between ERI at the end of follow-up and ERI at baseline

\begin{tabular}{lrrrrrr}
\hline Parameter & B & SE & t & p value & \multicolumn{2}{c}{$95 \%$ CIs } \\
\cline { 3 - 7 } & & & & & lower limit & upper limit \\
\hline Control treatment & 6.083 & 1.812 & 3.357 & 0.001 & 2.480 & 9.686 \\
Experimental treatment & 4.289 & 1.857 & 2.310 & 0.023 & 0.597 & -0.575 \\
Baseline ERI & -0.385 & 0.096 & -4.024 & 0.000 & -0.195 & \\
\hline
\end{tabular}

$\mathrm{R}$ square $=0.18$ (adjusted $\mathrm{R}$ square $=0.15)$.

creased on an average by $1.45 \mathrm{IU} / \mathrm{kg}^{*}$ g of $\mathrm{Hb} /$ week in the $\mathrm{ViE}$ group $(-11.32 \%)$ and increased by $0.53 \mathrm{IU} / \mathrm{kg}^{*} \mathrm{~g}$ of $\mathrm{Hb} /$ week in the control group $(+4.60 \%)$, with a mean net nonsignificant effect of $1.98 \mathrm{IU} / \mathrm{kg}^{*} \mathrm{~g}$ of Hb/week in favor of the ViE experimental group $(p=0.276)$. Following a pre-specified analysis, after taking into account the baseline ERI values, the differencebetween the experimental and control group became statistically significant $(\mathrm{p}=0.001)$.

Baseline ERI was a strong predictor of its changes during follow-up, with an inverse relationship $(\mathrm{p}=0.009)$ that was apparent only in the control group ( $\mathrm{R}$ square of 0.29 vs. 0.02 , in the control and experimental groups, respectively, $\mathrm{p}<0.001$; fig. 4 ).

\section{Multivariate Analysis}

The treatment group and baseline ERI values with their interaction explained $15 \%$ of the total variability of the ERI evolution during follow-up (table 3). As expected, baseline iron therapy was inversely related to ERI variations during follow-up, without reaching the critical $p$ values ( $b$ coefficient $-2.54, p=0.166$ ). Diabetic status $(\mathrm{p}=0.402)$, gender $(\mathrm{p}=0.257)$, ferritin $(\mathrm{p}=$ $0.167)$, C-reactive protein (CRP; $p=0.629)$, urea ratio $(\mathrm{p}=0.235)$ and PTH $(\mathrm{p}=0.212)$ values were also not significantly associated to ERI variations during follow-up. 


\section{Discussion}

We previously performed a randomized, cross-over, pilot study, which suggested a possible positive effect of the polysulfone ViE-bonded membrane dialyzer on ESA resistance after adjusting for baseline characteristics [11]. In particular, the positive effect was more likely in the patients with a higher ERI at baseline. Starting from this observation, we designed this larger, multicenter, randomized study.

Consistent with our previous findings [11], crude ERI changes during follow-up were not statistically different between the 2 groups. When adjusting the analysis for baseline ERI values, as suggested by our pilot study and foreseen by the protocol, the difference between the experimental and the control group became statistically significant, showing a positive effect of the $\mathrm{ViE}$-coated membrane on ERI. At the multivariate analysis, treatment group and baseline ERI with their interaction explained $15 \%$ of the total variability of ERI changes during follow-up.

A number of clinical studies [13-16] have evaluated the effects of ViE-bonded membranes with respect to oxidative stress, inflammation and ESA requirements with conflicting results.

Panichi et al. [13] performed a controlled trial of $62 \mathrm{HD}$ patients, who were randomized to either polysulfone + $\mathrm{ViE}$ or to polysulfone dialyzer alone for 6 months in a cross-over design. A significant decrease of ERI was observed only in the ViE period (from $10.3 \pm 2.2 \mathrm{IU}$-dl/ $\mathrm{kg} / \mathrm{g} \mathrm{Hb} /$ week at baseline to $9.2 \pm 1.7$ at 6 months). Interestingly, these changes in the anemia parameters went together with a significant decrease in interleukin-6 levels. Differing from our study, in this trial, low-flux membranes were used in both the experimental and control group.

Mandolfo et al. [14] also found a significant decrease in ERI after 6 months in a small, pilot, cross-over study of $16 \mathrm{HD}$ patients with central vein catheter, who were alternatively treated with a ViE-bonded dialyzer and a polyethersulfone membrane. Given this particular selection criteria, these patients were affected by more comorbidities and were hypo-responsive to ESA compared to our study population. This may have amplified the positive effect of the ViE membrane found by Mandolfo et al. [14] compared to our findings. A trend toward changes in protein glycoxidation, antioxidant and inflammatory markers was also observed.

Lines et al. [16] performed a large, randomized, clinical trial in the United Kingdom, which enrolled more than $200 \mathrm{HD}$ patients. Compared to polysulfone alone, overall only a positive trend was found in the ViE-bonded mem- brane on ERI after 12 months ( $p=0.08$ ). It is possible that the magnitude of this effect had been partially decreased by a higher percentage of diabetics and by a higher median unadjusted ESA doses at baseline in the experimental group. After stratifying the patients in tertiles of baseline ERI, a significant reduction was found only in those patients with the highest baseline ESA resistance who were receiving dialysis with the $\mathrm{ViE}$ membrane. Interestingly, the patients in the highest tertile of ERI had significantly higher CRP levels. However, in the patients dialyzing with the ViE membranes, the reduction of ERI was not mirrored by reductions in the CRP levels, and the change in ERI and CRP levels after 12 months were not correlated. This suggests that the reduction in ESA resistance seen in the high ERI group was not due to a reduction in systemic inflammation or maybe CRP testing was not sensitive enough. Of note, data from the Role of Hemodiafiltration (HDF) on ERI (REDERT) study showed that high-volume online HDF significantly reduced ERI during followup despite unchanged CRP levels [17].

Confirming the hypothesis that the 'anti-anemic' effect of efficient HD may be clearer in the patients who are ESA hypo-responsive, recently Marcelli et al. [18] found that the patients who benefit more in terms of anemia control are those with a high ERI at baseline also in the setting of HDF.

Sanaka et al. [15] were not able to confirm a positive effect of adding ViE to a high-flux polysulfone dialyzer. Considering the lower ESA doses in Japanese HD population in comparison to Western countries, this may have somehow reduced the statistical power of the study. Despite the large sample size $(n=300)$, the study had several drawbacks, such as a high number of dropouts, a very low number of analyzed patients per center in relation with the number of treatment groups, the lack of multivariate approach and the inflation derived from the use of multiple statistical tests.

Altogether, data from the literature seem to suggest that the higher ERI at baseline, the higher is the positive effect of the ViE-coated membrane. In our study, the effect of the ViE-bonded membrane in improving ESA responsiveness was evident in addition to the baseline levels of ERI.

Differing from our previous pilot study [11], in this trial, we did not find an association between PTH values and ERI. The same was true for the CRP values; however, these results are in agreement with the findings of Lines et al. [16].

Our study has a number of limitations. The relative small sample size and the higher-than-expected drop-out rate may have reduced the statistical power to detect a positive effect of the ViE-coated membrane at the unadjusted analysis. Another point of weakness is that the di- 
alysis technique was not homogenous between the 2 groups, since the experimental dialyzer was high-flux and the control one was low-flux. However, according to urea ratio values during follow-up, the dialysis dose was kept the same during the study in the 2 groups. Moreover, data in the literature do not support a significant role of high-flux dialysis on anemia improvement $[19,20]$.

In conclusion, this trial showed a positive effect of the ViE-coated membrane on ERI when taking the baseline ERI into account.

\section{Disclosure Statement}

Asaki Kaisei provided the ViE-coated filters of the study. Prof. F. Locatelli received fees from Asaki Kaisei as a speaker.

\section{Participating Centers}

Coordinating Center: Alessandro Manzoni Hospital, Lecco (F. Locatelli, S. Andrulli, L. Del Vecchio, S.M. Viganò); Ospedale Civile Augusto Murri, ASUR Marche Zona Territoriale 11, Fermo (M. Concetti, D. Garofalo, M. Di Cesare), Ospedale G.B. Morgagni, L. Pierantoni, Forlì (S. Urbini, L. Zambianchi); Ospedale Civile, Ivrea (TO) (F. Giacchino, F. Bonello), Presidio Ospedaliero Di Valle Camonica, Esine (BS) (R. Broccoli); Casa Sollievo della Sofferenza, San Giovanni Rotondo (FG) (F. Aucella, A. Gesuete); Ospedale SS Annunziata, Sassari (M. Cossu, G.F. Branca); Ospedale Misericordia, ASL Toscana SudEst, Grosseto (P. Conti, C. Gabbrielli); Ospedale di Senigallia, Senigallia (AN) (R. Boggi, L. Fattori); Ospedale Sant'Andrea, Roma (G. Punzo, L. Fazzari); Ospedale Campo di Marte, Lucca (A. Rosati, E. Parrini, D. Angelini); Ospedale Geriatrico, INRCA, Ancona (M. Peruzzini, S. Guerini, S. Baldini); Ospedale Forlanini, Roma (S. Di Giulio, M.P. Beraldi).

\section{References}

1 Locatelli F, Canaud B, Eckardt KU, Stenvinkel $\mathrm{P}$, Wanner $\mathrm{C}$, Zoccali C: Oxidative stress in end-stage renal disease: an emerging threat to patient outcome. Nephrol Dial Transplant 2003;18:1272-1280.

2 Del Vecchio L, Locatelli F, Carini M: What we know about oxidative stress in patients with chronic kidney disease on dialysis - clinical effects, potential treatment, and prevention. Semin Dial 2011;24:56-64.

3 Stenvinkel P, Heimbürger O, Paultre F, et al: Strong association between malnutrition, inflammation, and atherosclerosis in chronic renal failure. Kidney Int 1999;55:1899-1911.

4 Locatelli F, Andrulli S, Memoli B, et al: Nutritional-inflammation status and resistance to erythropoietin therapy in haemodialysis patients. Nephrol Dial Transplant 2006;21:991998.

5 Kato A, Odamaki M, Hishida A: Blood 8-hydroxy-2'-deoxyguanosine is associated with erythropoietin resistance in haemodialysis patients. Nephrol Dial Transplant 2003;18: 931-936.

6 Kalim S, Tamez H, Wenger J, et al: Carbamylation of serum albumin and erythropoietin resistance in end stage kidney disease. Clin J Am Soc Nephrol 2013;8:1927-1934.

7 Boaz M, Smetana S, Weinstein T, et al: Secondary prevention with antioxidants of cardiovascular disease in endstage renal disease (SPACE): randomised placebo-controlled trial. Lancet 2000;356:1213-1218.

8 Galli F, Rovidati S, Chiarantini L, Campus G, Canestrari F, Buoncristiani U: Bioreactivity and biocompatibility of a vitamin E-modified multi-layer hemodialysis filter. Kidney Int 1998;54:580-589.
9 Piroddi M, Pilolli F, Aritomi M, Galli F: Vita$\min \mathrm{E}$ as a functional and biocompatibility modifier of synthetic hemodialyzer membranes: an overview of the literature on vitamin E-modified hemodialyzer membranes. Am J Nephrol 2012;35:559-572.

10 Cruz DN, De Cal M, Garzotto F, et al: Effect of vitamin E-coated dialysis membranes on anemia in patients with chronic kidney disease: an Italian multicenter study. Int J Artif Organs 2008;31:545-552.

11 Andrulli S, Di Filippo S, Manzoni C, et al: Effect of synthetic vitamin E-bonded membrane on responsiveness to erythropoiesisstimulating agents in hemodialysis patients: a pilot study. Nephron Clin Pract 2010; 115:c82-c89.

12 Locatelli F, Covic A, Eckardt KU, et al; ERAEDTA ERBP Advisory Board: Anaemia management in patients with chronic kidney disease: a position statement by the Anaemia Working Group of European Renal Best Practice (ERBP). Nephrol Dial Transplant 2009; 24:348-354.

13 Panichi V, Rosati A, Paoletti S, et al: A vitamin E-coated polysulfone membrane reduces serum levels of inflammatory markers and resistance to erythropoietin-stimulating agents in hemodialysis patients: results of a randomized cross-over multicenter trial. Blood Purif 2011;32:7-14.

14 Mandolfo S, Corradi B, Bucci R, Farina M, Pilolli F, Galli F: Evaluation of the impact of a new synthetic vitamin E-bonded membrane on anemia and rHuEPO requirement in ESRD patients with central venous catheters: a pilot study. Int Urol Nephrol 2012;44:14931500 .
15 Sanaka T, Mochizuki T, Kinugasa E, et al: Randomized controlled open-label trial of vitamin E-bonded polysulfone dialyzer and erythropoiesis-stimulating agent response. Clin J Am Soc Nephrol 2013;8:969978 .

16 Lines SW, Carter AM, Dunn EJ, Lindley EJ, Tattersall JE, Wright MJ: A randomized controlled trial evaluating the erythropoiesis stimulating agent sparing potential of a vitamin E-bonded polysulfone dialysis membrane. Nephrol Dial Transplant 2014;29:649656

17 Panichi V, Scatena A, Rosati A, et al: Highvolume online haemodiafiltration improves erythropoiesis-stimulating agent (ESA) resistance in comparison with low-flux bicarbonate dialysis: results of the REDERT study. Nephrol Dial Transplant 2015;30: 682-689.

18 Marcelli D, Bayh I, Merello JI, et al: Dynamics of the erythropoiesis stimulating agent resistance index in incident hemodiafiltration and high-flux hemodialysis patients. Kidney Int 2016;90:192-202.

19 Locatelli F, Andrulli S, Pecchini F, et al: Effect of high-flux dialysis on the anaemia of haemodialysis patients. Nephrol Dial Transplant 2000;15:1399-1409.

20 Yokoyama H, Kawaguchi T, Wada T, et al; J-DOPPS Research Group: Biocompatibility and permeability of dialyzer membranes do not affect anemia, erythropoietin dosage or mortality in Japanese patients on chronic non-reuse hemodialysis: a prospective cohort study from the J-DOPPS II study. Nephron Clin Pract 2008;109:c100c108. 